\title{
Existence of two solutions for a fourth-order difference problem with $p(k)$ exponent
}

\author{
Mohsen Khaleghi Moghadam ${ }^{1} \cdot$ Yasser Khalili $^{1} \cdot$ Renata Wieteska $^{2}$
}

Received: 16 April 2019 / Accepted: 3 February 2020 / Published online: 15 February 2020

(c) The Author(s) 2020

\section{Abstract}

The existence of nontrivial solutions for a fourth-order discrete anisotropic boundary value problem involving the $p(k)$-Laplacian operator with the Dirichlet and the Neumann boundary value conditions is investigated. Variational approach based on a new critical point theorem is applied. An example is inserted to illustrate main results.

Keywords Discrete nonlinear boundary value problems $\cdot$ Nontrivial solution · Variational methods · Critical point theory

Mathematics Subject Classification 39A10 - 47A75 · 34B15

\section{Introduction}

The study of discrete boundary value problems has attracted intense research interests in the last decade. Modeling of certain nonlinear problems led to the rapid development of the theory of difference equations; see the monograph of [1]. Recently there have been an increasing interest to the existence and multiplicity results to boundary value problems for difference equations with the $p(k)$-Laplacian operator. Continuous versions of this kind of problem are known to be mathematical models of various phenomena arising in the study of elastic mechanics (see [24]), electrorheological fluids (see [22]), and image restoration (see [8]). Continuous variational anisotropic problems were started by Fan and Zhang in [9]. The research concerning the discrete fourth-order anisotropic problems have only been started,

$\bowtie \quad$ Renata Wieteska

renata.wieteska@p.lodz.pl

Mohsen Khaleghi Moghadam

m.khaleghi@sanru.ac.ir

Yasser Khalili

y.khalili@sanru.ac.ir

1 Department of Basic Sciences, Sari Agricultural Sciences and Natural Resources University, PO Box 578, Sari, Iran

2 The Center of Education of Mathematics and Physics Łodz University of Technology, Al. Politechniki 11, 90-924 Lodz, Poland 
see $[17,20]$ and have been followed by the other authors (see $[18,19])$, where known tools from the critical point theory are applied to prove the existence of solutions. Concerning the fourth-order problems with exponent variable we mainly follow [18].

The results on this topic are usually achieved by using fixed point theorems in cones (see [3] and references therein). Another tool in the study of nonlinear difference equations is the upper and lower solution method (see, for instance, [13] and references therein). It is well known that variational method and critical point theory are important tools to deal with the problems for differential equations. Recently, the existence and multiplicity of solutions for nonlinear discrete boundary value problems have been investigated by adopting variational methods (see $[2,12,15]$ ).

The main goal of this paper is to establish the existence of three solutions for the discrete anisotropic problem with a positive real parameter $\lambda$ on the form

$$
\left\{\begin{array}{l}
-\Delta^{2}\left(w(k-2) \phi_{p(k-2)}\left(\Delta^{2} u(k-2)\right)\right)+q(k)|u(k)|^{p(k)-2} u(k) \\
=\lambda f(k, u(k)), \quad k \in[1, T], \\
u(-1)=u(T+2)=\Delta u(-1)=\Delta u(T+1)=0,
\end{array}\right.
$$

where $T \geq 2$ is a fixed positive integer, $[1, T]$ is the discrete interval $\{1, \ldots, T\}, f$ : $[1, T+2] \times \mathbb{R} \rightarrow \mathbb{R}$ is a continuous function, $w:[-1, T+2] \rightarrow[1, \infty), p:[-1, T+2] \rightarrow$ $[2, \infty)$ and $q:[1, T+2] \rightarrow[1, \infty)$ are given functions, $\Delta u(k)=u(k+1)-u(k)$ is the forward difference operator and $\phi_{p(k)}$ is the so called the $p(k)$-Laplacian operator defined as $\phi_{p}(s)=|s|^{p-2} s$.

Directly from the definition of the forward difference operator we have

$$
\Delta^{2} u(k)=\Delta(\Delta u(k))=u(k+2)-2 u(k+1)+u(k) .
$$

Let us put

$$
\begin{array}{rlrl}
p^{+} & :=\max _{k \in[-1, T+2]} p(k), \quad p^{-}:=\min _{k \in[-1, T+2]} p(k), \\
q^{+} & :=\max _{k \in[1, T+2]} q(k), & w^{+}:=\max _{k \in[-1, T+2]} w(k), \\
\bar{q} & :=\sum_{k=1}^{T+2} q(k), & \bar{w} & :=\sum_{k=1}^{T+2} w(k-2) .
\end{array}
$$

Research concerning the discrete anisotropic problems of type (1) was initiated by Kone and Ouaro in [17] and by Mihăilescu, Rădulescu and Tersian in [20]. One can find in [4] and [6] further tools and ideas to study anisotropic discrete nonlinear problems. For the continuous counterpart of the fourth order discrete problems, one can see [23]. Also we may think of (1) as a discrete analogue of the fourth-order functional differential equation

$$
\left\{\begin{array}{l}
\frac{d^{2}}{d t^{2}}\left(w(t)\left|\frac{d^{2} u(t)}{d t^{2}}\right|^{p-2}\left(\frac{d^{2} u(t)}{d t^{2}}\right)\right)+q(t)|u(t)|^{p-2} u(t)=f(t, u(t)), \quad t \in(0,1), \\
u(0)=u(1)=u^{\prime \prime}(0)=u^{\prime \prime}(1)=0
\end{array}\right.
$$

A special case of the above equation is the equation

$$
\left\{\begin{array}{l}
u^{(4)}(t)=f(t, u(t)), \quad t \in(0,1) \\
u(0)=u(1)=u^{\prime \prime}(0)=u^{\prime \prime}(1)=0
\end{array}\right.
$$

which is used to model deformations of elastic beams [7,14,21].

The paper is arranged as follows. In Sect. 1 we recall the main tools. In Sect. 2, we introduce notations and provide several inequalities useful in our investigations. After variational 
framework in Sect. 3 we formulate and prove the main result and special case. Finally we present an example.

\section{Preliminaries}

Let $E$ be a real finite dimensional space. Given two Gâteaux differentiable mappings $\Phi, H$ : $E \rightarrow \mathbb{R}$ with derivatives $\varphi, h: E \rightarrow E^{*}$ we consider the following abstract equation

$$
\varphi(u)=h(u), \quad u \in E .
$$

We denote by $J: E \rightarrow \mathbb{R}$ the action functional connected with (2), i.e.

$$
J(u)=\Phi(u)-H(u) .
$$

Theorem 1 [10, Corollary 3.3] Assume that $X \subset E$ contains at least two points. Assume that $H$ and $\Phi$ are convex on $E$. Let there exist $u \in E, v \in X$ satisfying $\varphi(v)=h(u)$ such that $J(u) \leq \inf _{x \in X} J(x)$. Then $u$ is a critical point to $J$, and thus it solves (2).

We finish with a simple multiplicity result.

Theorem 2 [10, Theorem 3.4] Assume that $X \subset E$ contains at least two points. Assume that $H$ and $\Phi$ are convex on $E$. Let there exist $u \in E, v \in X$ satisfying $\varphi(v)=h(u)$ such that $J(u) \leq \inf _{x \in X} J(x)$. Then $u$ is a critical point to $J$, and thus it solves (2). If, moreover, $J$ is anti-coercive, then (2) has another solution different from $u$.

\section{Auxiliary inequalities}

Let us define the Euclidean space

$$
W:=\{u:[-1, T+2] \rightarrow \mathbb{R}: \Delta u(-1)=\Delta u(T+1)=u(-1)=u(T+2)=0\},
$$

which is equipped with the norm

$$
\|u\|:=\left(\sum_{k=1}^{T+2}\left(w(k-2)\left|\Delta^{2} u(k-2)\right|^{p^{-}}+q(k)|u(k)|^{p^{-}}\right)\right)^{1 / p^{-}} .
$$

Let us also define the following equivalent norms

$$
\begin{aligned}
\|u\|_{+} & :=\left(\sum_{k=1}^{T+2}\left(w(k-2)\left|\Delta^{2} u(k-2)\right|^{p^{+}}+q(k)|u(k)|^{p^{+}}\right)\right)^{1 / p^{+}}, \\
\|u\|_{\max } & :=\max _{k \in[-1, T+2]}|u(k)|
\end{aligned}
$$

and the Luxemburg norm

$$
\|u\|_{p(\cdot)}=\inf \left\{\mu>0: \sum_{k=1}^{T+2}\left(w(k-2)\left|\frac{\Delta^{2} u(k-2)}{\mu}\right|^{p(k-2)}+q(k)\left|\frac{u(k)}{\mu}\right|^{p(k)}\right) \leq 1\right\} .
$$

Note that there exists constant $L>0$ such that

$$
\|u\| \leq L\|u\|_{p(\cdot)} .
$$


Now we provide some inequalities used throughout the paper. Put

$$
K=(2 \max \{\bar{w}, \bar{q}\})^{\frac{p^{-}-p^{+}}{p^{+} p^{-}}} .
$$

Lemma 1 For every $u \in W$ we have what follows

(I1)

$$
\sum_{k=1}^{T+2}\left|\Delta^{2} u(k-2)\right|^{p} \leq 3^{p-1}\left(2^{p}+2\right) \sum_{k=1}^{T+2}|u(k)|^{p} \text { for any } p>1 .
$$

(I2)

$$
\left.\|u\| \leq(T+2)^{\frac{1}{p^{-}}-\frac{1}{p^{+}}}\left(w^{+} 3^{p^{-}-1}\left(2^{p^{-}}+2\right)+q^{+}\right)\right)^{\frac{1}{p^{-}}}\left(\sum_{k=1}^{T+2}|u(k)|^{p^{+}}\right)^{\frac{1}{p^{+}}} .
$$

$$
\|u\|_{+} \leq 2^{\frac{p^{+}-p^{-}}{p^{+} p^{-}}} K\|u\|
$$

(I4)

$$
\|u\|_{\max } \leq \frac{1}{4} \bar{w}^{\frac{2 p^{-}-1}{p^{-}}}\|u\| .
$$

Proof Relation (I1) is obtained by similar argument as in [11]. Using the inequality

$$
\left(\left|a_{1}\right|+\left|a_{2}\right|+\left|a_{3}\right|\right)^{p} \leq 3^{p-1}\left(\left|a_{1}\right|^{p}+\left|a_{2}\right|^{p}+\left|a_{3}\right|^{p}\right) \text { for any } p>1
$$

we have

$$
\begin{aligned}
\sum_{i=1}^{T+2}\left|\Delta^{2} u(i-2)\right|^{p} & =\sum_{i=1}^{T+2}|u(i)-2 u(i-1)+u(i-2)|^{p} \\
& \leq 3^{p-1}\left(2^{p}+2\right) \sum_{i=1}^{T+2}|u(i)|^{p} .
\end{aligned}
$$

By (I1) we get (I2) as follows

$$
\begin{aligned}
\|u\|^{p^{-}} & \leq w^{+} \sum_{i=1}^{T+2}\left(\left|\Delta^{2} u(i-2)\right|^{p^{-}}+q^{+}|u(i)|^{p^{-}}\right) \\
& \leq\left(w^{+} 3^{p^{-}-1}\left(2^{p^{-}}+2\right)+q^{+}\right) \sum_{i=1}^{T+2}|u(i)|^{p^{-}} .
\end{aligned}
$$

By the Hölder inequality we get

$$
\sum_{i=1}^{T+2}|u(i)|^{p^{-}} \leq(T+2)^{1-\frac{p^{-}}{p^{+}}}\left(\sum_{i=1}^{T+2}|u(i)|^{p^{+}}\right)^{\frac{p^{-}}{p^{+}}} .
$$

Hence by (4) and (5) now we have (I2). 
Relation (I3) is obtained by similar arguments as in [16]. By the weighted Hölder inequality and the Minkowski inequality we see that

$$
\begin{aligned}
\|u\|_{+}^{p^{+} \leq} & (\max \{\bar{w}, \bar{q}\})^{\frac{p^{-}-p^{+}}{p^{-}}} \\
& \times\left(\left(\sum_{k=1}^{T+2} w(k-2)\left|\Delta^{2} u(k-2)\right|^{p^{-}}\right)^{\frac{p^{+}}{p^{-}}}+\left(\sum_{k=1}^{T+2} q(k)|u(k)|^{p^{-}}\right)^{\frac{p^{+}}{p^{-}}}\right) \\
\leq & 2^{\frac{p^{+}-p^{-}}{p^{-}}} K^{p^{+}}\|u\|^{p^{+}} .
\end{aligned}
$$

To see (I4) note that for any $u \in W$ and for any $k \in[1, T+2]$ we have

$$
|u(k)|=\left|\sum_{i=1}^{k} \Delta u(i-1)\right| \leq \sum_{i=1}^{k}|\Delta u(i-1)|
$$

and

$$
|u(k)|=\left|\sum_{i=k+1}^{T+2} \Delta u(i-1)\right| \leq \sum_{i=k+1}^{T+2}|\Delta u(i-1)| .
$$

Combining the above inequalities by adding the left-hand sides and right-hand sides we obtain

$$
2|u(k)| \leq \sum_{i=1}^{T+2}|\Delta u(i-1)| .
$$

Since $u(-1)=0$, for any $k \in[-1, T+2]$, we get

$$
|u(k)| \leq \frac{1}{2} \sum_{i=1}^{T+2}|\Delta u(i-1)|
$$

Arguing as above, for any $k \in[1, T+2]$, we obtain

$$
|\Delta u(k-1)| \leq \frac{1}{2} \sum_{i=1}^{T+2}\left|\Delta^{2} u(i-2)\right| .
$$

Function $w$ has only positive value, so for any $u \in W$ by (6) and (7), respectively, we get

$$
|u(k)| \leq \frac{1}{2} \sum_{i=1}^{T+2} w(i-2)|\Delta u(i-1)| \text { for any } k \in[-1, T+2]
$$

and

$$
|\Delta u(k-1)| \leq \frac{1}{2} \sum_{i=1}^{T+2} w(i-2)\left|\Delta^{2} u(i-2)\right| \text { for any } k \in[1, T+2]
$$


Hence for any $k \in[-1, T+2]$ the Hölder inequality implies

$$
\begin{aligned}
|u(k)| & \leq \frac{1}{2} \max _{k \in[1, T+2]}|\Delta u(k-1)| \sum_{i=1}^{T+2} w(i-2) \\
& \leq \frac{1}{4} \bar{w} \sum_{i=1}^{T+2} w(i-2)\left|\Delta^{2} u(i-2)\right| \\
& \leq \frac{1}{4} \bar{w} \frac{2 p^{-}-1}{p^{-}}\left(\sum_{i=1}^{T+2} w(i-2)\left|\Delta^{2} u(i-2)\right|^{p^{-}}+q(i)|u(i)|^{p^{-}}\right)^{\frac{1}{p^{-}}} \\
& =\frac{1}{4} \bar{w}^{\frac{2 p^{-}-1}{p^{-}}}\|u\| .
\end{aligned}
$$

The proof of Lemma 1 is complete.

Let $\psi: W \rightarrow \mathbb{R}$ be given by the formula

$$
\psi(u):=\sum_{k=1}^{T+2}\left(w(k-2)\left|\Delta^{2} u(k-2)\right|^{p(k-2)}+q(k)|u(k)|^{p(k)}\right) .
$$

For any $u \in W$ the following properties hold (see [5]):

$$
\|u\|_{p(\cdot)}>1 \Rightarrow\|u\|_{p(\cdot)}^{p^{-}} \leq \psi(u) \leq\|u\|_{p(\cdot)}^{p^{+}} .
$$

Lemma 2 For all $u \in W$ we have

$$
\psi(u) \leq 2^{\frac{p^{+}-p^{-}}{p^{-}}} K^{p^{+}}\|u\|^{p^{+}}+\left(w^{+}+q^{+}\right)(T+2) .
$$

Proof Let $u \in W$. By a similar argument as in [16], we have

$$
\begin{aligned}
& \sum_{k=1}^{T+2} w(k-2)\left|\Delta^{2} u(k-2)\right|^{p(k-2)} \\
& \leq\left(\sum_{\left\{k \in[1, T+2]:\left|\Delta^{2} u(k-2)\right|<1\right\}}+\sum_{\left\{k \in[1, T+2]:\left|\Delta^{2} u(k-2)\right| \geq 1\right\}}\right) w(k-2)\left|\Delta^{2} u(k-2)\right|^{p^{-}} \\
& \quad+w(k-2)\left|\Delta^{2} u(k-2)\right|^{p^{+}} \\
& =\sum_{k=1}^{T+2} w(k-2)\left|\Delta^{2} u(k-2)\right|^{p^{+}} \\
& \quad+\sum_{\left\{k \in[1, T+2]:\left|\Delta^{2} u(k-2)\right|<1\right\}}\left(w(k-2)\left(\left|\Delta^{2} u(k-2)\right|^{p^{-}}-\left|\Delta^{2} u(k-2)\right|^{p^{+}}\right)\right) \\
& \leq \sum_{k=1}^{T+2} w(k-2)\left|\Delta^{2} u(k-2)\right|^{p^{+}}+w^{+}(T+2) .
\end{aligned}
$$

In the same manner we get

$$
\sum_{k=1}^{T+2} q(k)|u(k)|^{p(k)} \leq \sum_{k=1}^{T+2} q(k)|u(k)|^{p^{+}}+q^{+}(T+2) .
$$


Combining the above inequalities in view of (I4) we obtain inequality (9).

\section{Variational framework}

In this section we connect solutions to (1) with critical points of a suitably chosen action functional. Let

$$
F(k, t):=\int_{0}^{t} f(k, \xi) d \xi \text { for every }(k, t) \in[1, T] \times \mathbb{R} .
$$

Let $\lambda>0$ be fixed. We consider a functional $I_{\lambda}: W \rightarrow \mathbb{R}$ defined by

$$
I_{\lambda}(u):=\sum_{k=1}^{T+2}\left(\frac{w(k-2)}{p(k-2)}\left|\Delta^{2} u(k-2)\right|^{p(k-2)}+\frac{q(k)}{p(k)}|u(k)|^{p(k)}\right)-\lambda \sum_{k=1}^{T+2} F(k, u) .
$$

Put

$$
\begin{aligned}
\Phi(u) & :=\sum_{k=1}^{T+2}\left(\frac{w(k-2)}{p(k-2)}\left|\Delta^{2} u(k-2)\right|^{p(k-2)}+\frac{q(k)}{p(k)}|u(k)|^{p(k)}\right), \\
H(u) & :=\sum_{k=1}^{T+2} F(k, u(k)) .
\end{aligned}
$$

Then $I_{\lambda}=\Phi-\lambda H$.

Applying twice, for the functional $\Phi$, the summation by parts formula and use the conditions $\Delta v(-1)=v(0)=\Delta v(T+1)=v(T+2)=0$ we can see that

$$
\Phi^{\prime}(u)(v)=\sum_{k=1}^{T+2}\left(\Delta^{2}\left(w(k-2) \phi_{p(k-2)}\left(\Delta^{2} u(k-2)\right)\right) v(k)+q(k) \phi_{p(k)}(u(k)) v(k)\right)
$$

for all $u, v \in W$. Therefore $\Phi$ is of class $C^{1}$ on $W$.

The derivative of $H$ reads

$$
H^{\prime}(u)(v)=\sum_{k=1}^{T+2} f(k, u(k)) v(k)
$$

for all $u, v \in W$. Therefore $H$ is of class $C^{1}$ on $W$. Hence $I_{\lambda}$ is of class $C^{1}$ on $W$.

Lemma 3 The functional $\Phi$ is coercive.

Proof To prove the coercivity of $\Phi$ note that for $\|u\|$ large as well , $\|u\|_{p(.)}$ is large enough, so by (8) and (3) we get

$$
\Phi(u) \geq \frac{\psi(u)}{p^{+}} \geq \frac{\|u\|_{p(.)}^{p^{-}}}{p^{+}} \geq \frac{\|u\|^{p^{-}}}{p^{+} L^{p^{-}}} .
$$

Hence, as $\|u\| \rightarrow+\infty$, we can conclude that $\Phi(u) \rightarrow+\infty$.

Lemma 4 The function $u \in W$ is a critical point of $I_{\lambda}$ in $W$ iff $u$ is a solution of problem (1). 
Proof First, let $\bar{u}$ be a critical point of $I_{\lambda}$ in $W$. Then for all $v \in W, I_{\lambda}^{\prime}(\bar{u})(v)=0$ and $\Delta \bar{u}(-1)=\Delta \bar{u}(T+1)=\bar{u}(-1)=\bar{u}(T+2)=0$. Thus, for every $v \in W$, taking twice summation by parts and taking $\Delta v(-1)=\Delta v(T+1)=v(-1)=v(T+2)=0$ into account we have

$$
\begin{aligned}
0= & I_{\lambda}^{\prime}(\bar{u})(v) \\
= & \sum_{k=1}^{T+2}\left(\Delta^{2}\left(w(k-2) \phi_{p(k-2)}\left(\Delta^{2} \bar{u}(k-2)\right)\right) v(k)+q(k) \phi_{p(k)}(\bar{u}(k)) v(k)\right) \\
& -\lambda \sum_{k=1}^{T+2} f(k, \bar{u}(k)) v(k) .
\end{aligned}
$$

Since $v \in W$ is arbitrary we get

$$
\begin{aligned}
& -\Delta^{2}\left(w(k-2)\left|\Delta^{2} \bar{u}(k-2)\right|^{p(k-2)-2} \Delta^{2} \bar{u}(k-2)\right)+q(k)|\bar{u}(k)|^{p(k)-2} \bar{u}(k) \\
& =\lambda f(k, \bar{u}(k)),
\end{aligned}
$$

for every $k \in[1, T]$. Therefore, $\bar{u}$ is a solution of (1). We conclude that every critical point of $I_{\lambda}$ in $W$ is a solution of problem (1).

We will employ the following assumptions.

(H1) $B^{\infty}:=\min _{k \in[1, T]} \lim \sup _{x \rightarrow+\infty} \frac{F(k, x)}{|x|^{p^{+}}}>0$ and $x \rightarrow F(k, x)$ is convex on $\mathbb{R}$ for all $k \in[1, T]$.

Let

$$
\lambda^{*}:=\frac{2^{\frac{p^{+}-p^{-}}{p^{-}}} K^{p^{+}}}{p^{-} B^{\infty}\left(3 p^{--1} w^{+}\left(2^{p^{-}}+2\right)+q^{+}\right)^{-\frac{p^{+}}{p^{-}}}(T+2)^{1-\frac{p^{+}}{p^{-}}}} .
$$

Lemma 5 Assume that (H1) is satisfied. Then for any $\lambda>\lambda^{*}$ the functional $I_{\lambda}$ is anti-coercive, i.e. $I_{\lambda}(u) \rightarrow-\infty$ as $\|u\| \rightarrow+\infty$.

Proof Let us fix $\lambda>\lambda^{*}$. Taking $\lim \sup _{x \rightarrow+\infty} \frac{F(k, x)}{|x|^{p^{+}}} \geq B^{\infty}$ we will find $\delta>0$ with $F(k, x) \geq B^{\infty}|x|^{p^{+}}$for any $k \in[1, T]$ and for any $x \in \mathbb{R}$ with $|x|>\delta$.

For $\|u\|$ sufficiently large, by (I3) we get

$$
\begin{aligned}
-\lambda \sum_{k=1}^{T+2} F(k, u(k)) & \leq-\lambda B^{\infty} \sum_{k=1}^{T+2}|u(k)|^{p^{+}} \\
& \leq-\lambda B^{\infty}\left(3^{p^{-}-1} w^{+}\left(2^{p^{-}}+2\right)+q^{+}\right)^{-\frac{p^{+}}{p^{-}}}(T+2)^{1-\frac{p^{+}}{p^{-}}}\|u\|^{p^{+}}
\end{aligned}
$$

and by (9) we have

$$
\Phi(u) \leq \frac{\psi(u)}{p^{-}} \leq \frac{1}{p^{-}}\left(2^{\frac{p^{+}-p^{-}}{p^{-}}} K^{p^{+}}\|u\|^{p^{+}}+\left(w^{+}+q^{+}\right)(T+1)\right) .
$$


Hence, by (12) and (13) we obtain

$$
\begin{aligned}
I_{\lambda}(u)= & \Phi(u)-\lambda \sum_{k=1}^{T+1} F(k, u(k)) \\
\leq & \frac{1}{p^{-}}\left(2^{\frac{p^{+}-p^{-}}{p^{-}}} K^{p^{+}}\|u\|^{p^{+}}+\left(w^{+}+q^{+}\right)(T+1)\right) \\
& -\lambda B^{\infty}\left(3^{p^{-}-1} w^{+}\left(2^{p^{-}}+2\right)+q^{+}\right)^{-\frac{p^{+}}{p^{-}}}(T+2)^{1-\frac{p^{+}}{p^{-}}\|u\|^{p^{+}}} \\
= & \frac{1}{p^{-}}\left(w^{+}+q^{+}\right)(T+1) \\
& +\left(\frac{1}{p^{-}} 2^{\frac{p^{+}-p^{-}}{p^{-}}} K^{p^{+}}-\lambda B^{\infty}\left(3^{p^{-}-1} w^{+}\left(2^{p^{-}}+2\right)+q^{+}\right)^{-\frac{p^{+}}{p^{-}}}(T+2)^{1-\frac{p^{+}}{p^{-}}}\right)\|u\|^{p^{+}} \\
= & \frac{1}{p^{-}}\left(w^{+}+q^{+}\right)(T+1) \\
& +B^{\infty}\left(3^{p^{-}-1} w^{+}\left(2^{p^{-}}+2\right)+q^{+}\right)^{-\frac{p^{+}}{p^{-}}}(T+2)^{1-\frac{p^{+}}{p^{-}}}\left(\lambda^{*}-\lambda\right)\|u\|^{p^{+}} .
\end{aligned}
$$

So taking $B^{\infty}>0$, one can conclude that $I_{\lambda}(u) \rightarrow-\infty$ as $\|u\| \rightarrow+\infty$.

\section{Main results}

We state our main result as follows.

Theorem 3 Suppose that assumption (H1) is satisfied. Then, for any $\left.\lambda \in \Lambda_{1}:=\right] \lambda^{*},+\infty[$ problem (1) has at least one nontrivial solution.

Proof Take $E=W$ and $\Phi, H$ as in (10) and (11). Note that these are convex $C^{1}$ functionals. By Lemma 5 functional $I_{\lambda}$ for any $\lambda \in \Lambda_{1}$ is anticoercive and since it is $C^{1}$ functional in a finite dimensional $W$, so it has obviously at least one maximizer which is a critical point of $I_{\lambda}$. Thus by Lemma 4 the problem (1) has at least one nontrivial solution.

Let

(H2) $B_{0}:=\max _{k \in[1, T+2]} \liminf \operatorname{in}_{x \rightarrow 0} \frac{F(k, x)}{|x|^{p^{-}+1}}<\infty$.

Put

$$
\lambda^{* *}=\frac{4 L^{-p^{-}-2}}{\left(p^{-}+1\right) B_{0} \bar{w}^{\frac{2 p^{-}-1}{p^{-}}}} .
$$

Theorem 4 Suppose that the assumption $(H 2)$ is satisfied. Then for any $\lambda \in] 0, \lambda^{* *}[$ problem (1) has at least one nontrivial solution.

Proof From (H2), one can conclude that

$$
f(k, x) \leq\left(p^{-}+1\right) B_{0}|x|^{p^{-}} \text {for all }|x| \leq L \text { and all } k \in[1, T+2],
$$

where $L>0$ satisfies (3). Let us define a set $D \subset E$ by

$$
D=\left\{x \in E:\|x\|_{p(.)} \leq L\right\} .
$$


Fix $\lambda \in] 0, \lambda^{* *}\left[\right.$. We shall apply Theorem 1 . The functional $I_{\lambda}$ is continuous and the subset $D$ is closed and bounded, therefore there exists a minimum of $I_{\lambda}$ over $D$, which we denote by $x_{0}$, so

$$
\left\|x_{0}\right\|_{p(.)}<L .
$$

Consider on the space $E$ the following Dirichlet problem

$$
\left\{\begin{array}{l}
-\Delta^{2}\left(w(k-2) \phi_{p(k-2)}\left(\Delta^{2} x(k-2)\right)+q(k)|x(k)|^{p(k)-2} x(k)\right. \\
\quad=\lambda f\left(k, x_{0}(k)\right), k \in[1, T] \\
x(-1)=x(T+2)=\Delta x(-1)=\Delta x(T+1)=0 .
\end{array}\right.
$$

The energy functional $J: E \rightarrow \mathbb{R}$ corresponding to (16) is on the form

$$
J(x)=\Phi(x)-\lambda H\left(x_{0}\right) .
$$

From Lemma 3 the functional $J$ is coercive. It is also $C^{1}$ and strictly convex, so problem (16) is uniquely solvable by some $v \in E$. We shall prove that $v \in D$. If $\|v\|<1$ the conclusion is immediate. Suppose $\|v\| \geq 1$. Multiplying

$$
-\Delta^{2}\left(w(k-2) \phi_{p(k-2)}\left(\Delta^{2} v(k-2)\right)+q(k)|v(k)|^{p(k)-2} v(k)=\lambda f\left(k, x_{0}(k)\right)\right.
$$

by $v$ and summing from 1 to $T+2$ we have what follows

$$
\begin{aligned}
& \sum_{k=1}^{T+2}\left(\Delta^{2}\left(w(k-2) \phi_{p(k-2)}\left(\Delta^{2} v(k-2)\right)\right) v(k)+q(k) \phi_{p(k)}(v(k)) v(k)\right) \\
& \quad=\lambda \sum_{k=1}^{T+2} f\left(k, x_{0}(k)\right) v(k) .
\end{aligned}
$$

Taking twice summation by parts and taking $\Delta v(-1)=\Delta v(T+1)=v(-1)=v(T+2)=0$ into account, one has

$$
\psi(v)=\lambda \sum_{k=1}^{T+2} f\left(k, x_{0}(k)\right) v(k) .
$$

By (8) we see

$$
\|v\|_{p(.)}^{p^{-}} \leq \psi(v) .
$$

On the other hand from (14), (3), (15), (14), (3), respectively, we obtain

$$
\begin{aligned}
& \lambda \sum_{k=1}^{T+2} f\left(k, x_{0}(k)\right) v(k) \leq \lambda \sum_{k=1}^{T+2}\left(p^{-}+1\right) B_{0}\left|x_{0}(k)\right|^{p^{-}} v(k) \\
& \quad \leq\left(p^{-}+1\right) \lambda B_{0}\|v(k)\|_{\max } \sum_{k=1}^{T+2}\left|x_{0}\right|^{p^{-}} \leq\left(p^{-}+1\right) \lambda B_{0}\|v(k)\|_{\max }\left\|x_{0}\right\|^{p^{-}} \\
& \quad \leq\left(p^{-}+1\right) \lambda B_{0} L^{p^{-}}\left\|x_{0}\right\|_{p(.)}^{p^{-}}\|v\|_{\max } \leq\left(p^{-}+1\right) \lambda B_{0} L^{2 p^{-}}\|v\|_{\max } \\
& \quad \leq\left(p^{-}+1\right) \lambda B_{0} L^{2 p^{-}} \frac{1}{4} \bar{w} \frac{2 p^{-}-1}{p^{-}}\|v\| \leq \frac{1}{4}\left(p^{-}+1\right) \lambda B_{0} L^{2 p^{-}} \bar{w}^{\frac{2 p^{-}-1}{p^{-}}} L\|v\|_{p(.)} \\
& \quad=\frac{1}{4}\left(p^{-}+1\right) \lambda B_{0} L^{2 p^{-}+1} \frac{2 p^{-}-1}{w^{-}}\|v\|_{p(.)} .
\end{aligned}
$$


So

$$
\|v\|_{p(.)}^{p^{-}} \leq \frac{1}{4}\left(p^{-}+1\right) \lambda B_{0} L^{2 p^{-}+1} \bar{w}^{\frac{2 p^{-}-1}{p^{-}}}\|v\|_{p(.)}
$$

and then

$$
\|v\|_{p(.)}^{p^{-}-1} \leq \frac{1}{4}\left(p^{-}+1\right) \lambda B_{0} L^{2 p^{-}+1} \bar{w}^{\frac{2 p^{-}-1}{p^{-}}} .
$$

Hence for any $\lambda<\lambda^{* *}$ we obtain

$$
\|v\|_{p(.)}^{p^{-}-1} \leq \frac{1}{4}\left(p^{-}+1\right) \lambda B_{0} L^{2 p^{-}+1} \bar{w}^{\frac{2 p^{-}-1}{p^{-}}}<L^{p^{-}-1} .
$$

Therefore, $v \in D$. Applying Theorem 1 we see that problem (1) has at least one nontrivial solution.

Theorem 5 Suppose that the assumptions (H1)-(H2) are satisfied. Let $\lambda^{*}<\lambda^{* *}$. Then for any $\lambda \in] \lambda^{*}, \lambda^{* *}[$ problem (1) has at least two nontrivial solutions.

Proof To prove our result it is enough to apply Theorem 3 and Theorem 4. By Theorem 3, $u$ is a critical point to $J$, and thus it solves (1). Also $J$ is anti-coercive, then (1) has another solution different from $u$.

Corollary 1 By Theorem 5, if the conditions $B_{\infty}=+\infty$ and $B_{0}=0$ hold. Then for any $\lambda>0$ problem (1) has at least two nontrivial solutions.

Finally, we present a special case of our main result with an example.

Theorem 6 Let $f$ be a continuous function on $\mathbb{R}$ and let $F(x)=\int_{0}^{x} f(\xi) d \xi$ be a convex function on $\mathbb{R}$ satisfying following conditions

$$
\limsup _{x \rightarrow+\infty} \frac{F(x)}{|x|^{p^{+}}}=+\infty, \quad \liminf _{x \rightarrow 0} \frac{F(x)}{|x|^{p^{-}+1}}=0 .
$$

Then, for each $\lambda>0$ the problem

$$
\left\{\begin{array}{l}
-\Delta^{2}\left(\left|\Delta^{2} u(k-2)\right|^{p(k-2)-2} \Delta^{2} u(k-2)\right)+|u(k)|^{p(k)-2} u(k) \\
=\lambda f(u(k)), k \in[1, T] \\
u(-1)=u(T+2)=\Delta u(-1)=\Delta u(T+1)=0,
\end{array}\right.
$$

admits at least two solutions.

Example 1 Let $T=4, p(k)=\frac{6 k^{2}-34 k+54}{k^{2}-6 k+10}$, hence $p^{+}=p(4)=7, p^{-}=p(2)=5$. Let $f(t)=\sum_{n=4}^{\infty} a_{n} t^{2 n+1}, a_{n} \in \mathbb{R}^{+}$and $F(t)=\sum_{n=4}^{\infty} a_{n} \frac{t^{2 n+2}}{2 n+2}$. Conditions (17) are fulfilled, so by Theorem 6 , for each $\lambda>0$ problem (18) admits at least two solutions.

Acknowledgements The authors are thankful to anonymous referee for helpful suggestions and comments. The authors express their gratitude to Professor Marek Galewski for his helpful suggestions.

Funding This work was supported by Sari agricultural sciences and natural resources university (Grant number 03-1397-07).

Open Access This article is licensed under a Creative Commons Attribution 4.0 International License, which permits use, sharing, adaptation, distribution and reproduction in any medium or format, as long as you give appropriate credit to the original author(s) and the source, provide a link to the Creative Commons licence, and indicate if changes were made. The images or other third party material in this article are included in the article's Creative Commons licence, unless indicated otherwise in a credit line to the material. If material is not included in the article's Creative Commons licence and your intended use is not permitted by statutory regulation or exceeds the permitted use, you will need to obtain permission directly from the copyright holder. To view a copy of this licence, visit http://creativecommons.org/licenses/by/4.0/. 


\section{References}

1. Agarwal, R.P.: Difference Equations and Inequalities: Theory. Methods and Applications. Marcel Dekker, New York (2000)

2. Avci, M.: Existence results for anisotropic discrete boundary value problems. Electron. J. Differ. Equ. 2016(148), 1-11 (2016)

3. Avery, R., Henderson, J.: Existence of three positive pseudo-symmetric solutions for a one dimensional discrete p-Laplacian. J. Differ. Equ. Appl. 10, 529-539 (2004)

4. Bonanno, G., Candito, P., D’Aguì, G.: Positive solutions for a nonlinear parameter-depending algebraic system. Electron. J. Differ. Equ. 2015(17), 1-14 (2015)

5. Bonanno, G., Jebelean, P., Serban, C.: Three solutions for discrete anisotropic periodic and neumann problems. Dyn. Syst. Appl. 22, 183-196 (2013)

6. Candito, P., D’Aguì, G., O’Regan, D.: Constant sign solutions for parameter-dependent superlinear second-order difference equations. J. Differ. Equ. Appl. 218, 649-659 (2015)

7. Chang, K.C.: Infinite Dimensional Morse Theory and Multiple Solution Problems. Birkhäuser, Boston (1993)

8. Chen, Y., Levine, S., Rao, M.: Variable exponent, linear growth functionals in image processing. SIAM J. Appl. Math. 66(4), 1383-1406 (2006)

9. Fan, X.L., Zhang, H.: Existence of solutions for $\mathrm{p}(\mathrm{x})$-Lapacian Dirichlet problem. Nonlinear Anal. Theory Methods Appl. 52 8(A), 1843-1852 (2003)

10. Galewski, M., Galewska, E.: On a new critical point theorem and some applications to discrete equations. Opuscula Math. 34(4), 725-732 (2014)

11. Galewski, M., Wieteska, R.: Existence and multiplicity of positive solutions for discrete anisotropic equations. Turk. J. Math. 38, 297-310 (2014)

12. Heidarkhani, S., Khaleghi Moghadam, M.: Existence of Three solutions for Perturbed nonlinear difference equations. Opuscula Math. 344, 747-761 (2014)

13. Jiang, D., Chu, J., O’Regan, D., Agarwal, R.P.: Positive solutions for continuous and discrete boundary value problems to the one-dimensional $p$-Laplacian. Math. Inequal. Appl. 7, 523-534 (2004)

14. Khaleghi Moghadam, M.: Existence of a non-trivial solution for fourth-order elastic beam equations involving Lipschitz non-linearity. Cogent Math. 3, 1226040 (2016)

15. Khaleghi Moghadam, M., Henderson, J.: Triple solutions for a dirichlet boundary value problem involving a perturbed discrete $p(k)$-Laplacian operator. Open Math. J. 15, 1075-1089 (2017)

16. Khaleghi Moghadam, M., Wieteska, R.: Existence and uniqueness of positive solution for nonlinear difference equations involving $p(k)$-Laplacian operator. An. Stiint. Univ. Ovidius Constanta Ser. Mat. 27(1), 141-167 (2019)

17. Kone, B., Ouaro, S.: Weak solutions for anisotropic discrete boundary value problems. J. Differ. Equ. Appl. 17(10), 1537-1547 (2011)

18. Leszczyński, M.: Fourth-order discrete anisotropic boundary-value problems. Electron. J. Differ. Equ. 2015(238), 1-14 (2015)

19. Liu, X., Zhang, Y., Shi, H.: Existence and nonexistence results for a fourth-order discrete Dirichlet boundary value problem. Hacet. J. Math. Stat. 44(4), 855-866 (2015)

20. Mihăilescu, M., Rădulescu, V., Tersian, S.: Eigenvalue problems for anisotropic discrete boundary value problems. J. Differ. Equ. Appl. 15, 557-567 (2009)

21. Rabinowitz, P.H.: Minimax Methods in Critical Point Theory with Applications to Differential Equations. American Mathematical Society, Providence (1986)

22. Ružička, M.: Electrorheological Fluids: Modelling and Mathematical Theory. Lecture Notes in Mathematics, vol. 1748. Springer, Berlin (2000)

23. Wang, F., Avci, M., An, Y.: Existence of solutions for fourth order elliptic equations of Kirchhoff type. J. Math. Anal. Appl. 409(1), 140-146 (2014)

24. Zhikov, V.V.: Averaging of functionals of the calculus of variations and elasticity theory. Math. USSR Izv. 29, 33-66 (1987)

Publisher's Note Springer Nature remains neutral with regard to jurisdictional claims in published maps and institutional affiliations. 